\title{
An Attention-Driven Model for Grouping Similar Images with Image Retrieval Applications
}

\author{
Oge Marques, ${ }^{1}$ Liam M. Mayron, ${ }^{1}$ Gustavo B. Borba, ${ }^{2}$ and Humberto R. Gamba ${ }^{2}$ \\ ${ }^{1}$ Department of Computer Science and Engineering, Florida Atlantic University, Boca Raton, FL 33431-0991, USA \\ ${ }^{2}$ Programa de Pós-Graduação em Engenharia Elétrica e Informática Industrial, Universidade Tecnológica Federal do Paraná (UTFPR), \\ Curitiba, Paraná 80230-901, Brazil
}

Received 1 December 2005; Revised 3 August 2006; Accepted 26 August 2006

Recommended by Gloria Menegaz

\begin{abstract}
Recent work in the computational modeling of visual attention has demonstrated that a purely bottom-up approach to identifying salient regions within an image can be successfully applied to diverse and practical problems from target recognition to the placement of advertisement. This paper proposes an application of a combination of computational models of visual attention to the image retrieval problem. We demonstrate that certain shortcomings of existing content-based image retrieval solutions can be addressed by implementing a biologically motivated, unsupervised way of grouping together images whose salient regions of interest (ROIs) are perceptually similar regardless of the visual contents of other (less relevant) parts of the image. We propose a model in which only the salient regions of an image are encoded as ROIs whose features are then compared against previously seen ROIs and assigned cluster membership accordingly. Experimental results show that the proposed approach works well for several combinations of feature extraction techniques and clustering algorithms, suggesting a promising avenue for future improvements, such as the addition of a top-down component and the inclusion of a relevance feedback mechanism.
\end{abstract}

Copyright ( 2007 Hindawi Publishing Corporation. All rights reserved.

\section{INTRODUCTION}

The dramatic growth in the amount of digital images available for consumption and the popularity of inexpensive hardware and software for acquiring, storing, and distributing images have fostered considerable research activity in the field of content-based image retrieval (CBIR) [1] during the past decade [2,3]. Simply put, in a CBIR system users search the image repository providing information about the actual contents of the image, which is often done using another image as an example. A content-based search engine translates this information in some way as to query the database (based on previously extracted and stored indexes) and retrieve the candidates that are more likely to satisfy the user's request.

In spite of the large number of related papers, prototypes, and several commercial solutions, the CBIR problem has not been satisfactorily solved. Some of the open problems include the gap between the image features that can be extracted using image processing algorithms and the semantic concepts to which they may be related (the well-known semantic gap problem [4-6], which can often be translated as "the discrepancy between the query a user ideally would and the one it actually could submit to an information retrieval system" [7]), the lack of widely adopted testbeds and benchmarks $[8,9]$, and the inflexibility and poor functionality of most existing user interfaces, to name just a few.

Some of the early CBIR solutions extract global features and index an image based on them. Other approaches take into account the fact that, in many cases, users are searching for regions or objects of interest as opposed to the entire picture. This has led to a number of proposed solutions that do not treat the image as a whole, but rather deal with portions (regions or blobs) within an image, such as $[10,11]$, or focus on objects of interest, instead [12]. The object-based approach for the image retrieval problem has grown to become an area of research referred to as object-based image retrieval (OBIR) in the literature [12-14].

Object- and region-based approaches usually must rely on image segmentation algorithms, which leads to a number of additional problems. More specifically, they must employ strong segmentation - "a division of the image data into regions in such a way that region $T$ contains the pixels of the silhouette of object $O$ in the real world and nothing else" [3], which is unlikely to succeed for broad image domains. A frequently used alternative to strong segmentation is weak segmentation, in which "region $T$ is within bounds of object 
$O$, but there is no guarantee that the region covers all of the object's area" [3], leading to imperfect-but usually acceptable for image retrieval purposes-results.

The limited success of CBIR solutions is further compounded by the fact that supervised learning (and, optionally, associated image annotation) — which could lead to improved efficiency and more accurate recognition results-is a subjective, usually domain-dependent, time-consuming, and expensive process, which makes it unrealistic for most realworld applications.

In this paper a new model to extract regions of interest (ROIs) within an image is proposed. The architecture was inspired by the success of a recently developed computational model of human visual attention [15], which provides important cues about the location of the most salient ROIs within an image. These ROIs, once extracted, are then indexed (based on their features) and clustered with other similar ROIs that may have appeared in other images.

This paper is structured as follows: Section 2 reviews relevant previous work in the fields of CBIR and computational modeling of human visual attention. Section 3 presents an overview of the proposed model and explains in detail its key features and components. Section 4 describes experiments performed with the current version of the prototype and discusses relevant results. Finally, Section 5 contains concluding remarks and directions for future work.

\section{BACKGROUND AND CONTEXT}

This section reviews relevant previous work on two separate areas brought together by the proposed model: CBIR systems and computational models of visual attention. We discuss the composition of a traditional CBIR system and how and where the proposed work fits within that context. Additionally, we present background on computational models of visual attention, particularly the model proposed by Itti et al. [15] and one proposed by Stentiford [16].

\subsection{CBIR systems}

CBIR refers to the retrieval of images according to their content, as opposed to the use of keywords. The purpose of a CBIR system is to retrieve all the images that are relevant to a user query while retrieving as few nonrelevant images as possible. Similarly to its text-based counterpart, an image retrieval system must be able to interpret the contents of the documents (images) in a collection and rank them according to a degree of relevance to the user query. The interpretation process involves extracting semantic information from the documents (images) and using this information to match the user's needs [17].

Figure 1 shows a block diagram of a generic CBIR system, whose main components are the following [1].

(i) User interface: friendly graphical user interface (GUI) that allows the user to interactively query the database, browse the results, and view the retrieved images.

(ii) Query/search engine: collection of algorithms responsible for searching the database according to the parameters provided by the user.

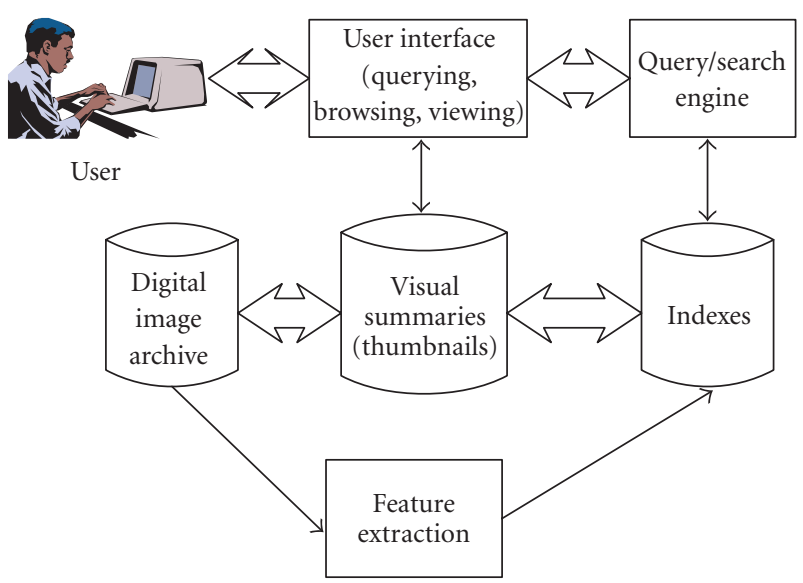

FIgURE 1: A generic CBIR architecture (adapted from [1]).

(iii) Digital image archive: repository of digitized (and usually compressed) images.

(iv) Visual summaries: representation of image in a concise way, such as thumbnails.

(v) Indexes: pointers to images.

(vi) Feature extraction: process of extracting (usually lowlevel) features from the raw images and using them to build the corresponding indexes.

Feature extraction is typically an offline process. Once it has been performed, the database will contain the image files themselves, possible simplified representations of each image file, and a collection of indexes that act as pointers to the corresponding images [1].

The online interaction between a user and a CBIR system is represented on the upper half of the diagram in Figure 1. The user expresses his query using a GUI. That query is translated and a search engine looks for the index that corresponds to the desired image. The results are sent back to the user in a way that should allow easy browsing, viewing, and possible refinement of the query based on the partial results [1].

Most CBIR systems allow searching the visual database contents in several different ways, either alone or combined [1].

(i) Interactive browsing: convenient to leisure users who may not have specific ideas about the images they are searching for. Clustering techniques can be used to organize visually similar images into groups and minimize the number of undesired images shown to the user.

(ii) Navigation with customized categories: leisure users often find it very convenient to navigate through a subject hierarchy to get to the target subject and then browse or search that limited subset of images.

(iii) Query by $X$, where " $X$ " can be [18]

(1) an image example: several systems allow the user to specify an image (virtually anywhere in the Internet) as an example and search for the images 
that are most similar to it, presented in decreasing order of similarity score. It is considered to be the most classical paradigm of image search,

(2) a visual sketch: some systems provide users with tools that allow drawing visual sketches of the image they have in mind. Users are also allowed to specify different weights for different features,

(3) specification of visual features: direct specification of visual features (e.g., color, texture, shape, and motion properties) is possible in some systems and might appeal to more technical users,

(4) a keyword or complete text: some image retrieval systems rely on keywords entered by the user and search for visual information that has been previously annotated using that (set of) keyword(s),

(5) a semantic class: where users specify (or navigate until they reach) a category in a preexisting subject hierarchy.

Progress in CBIR has been fostered by recent research results in many fields, including (text-based) information retrieval, image processing and computer vision, visual data modeling and representation, human-computer interaction, multidimensional indexing, human visual perception, pattern recognition, multimedia database organization, among others [1].

CBIR is essentially different from the general image understanding problem. More specifically, it is usually sufficient that a CBIR system retrieves similar-in some userdefined sense-images, without fully interpreting its contents. CBIR provides a new framework and additional challenges for computer vision solutions, such as the large data sets involved, the inadequacy of strong segmentation, the key role played by color, and the importance of extracting features and using similarity measures that strike a balance between invariance and discriminating power [3].

Ultimately, effective CBIR systems will overcome two great challenges: the sensory gap and the semantic gap. The sensory gap is "the gap between the object in the world and the information in a (computational) description derived from a recording of that scene" [3]. The sensory gap is comparable to the general problem of vision: how one can make sense of a 3D scene (and its relevant objects) from (one of many) 2D projections of that scene. CBIR systems usually deal with this problem by eliminating unlikely hypotheses, much the same way as the human visual system (HVS) does, as suggested by Helmholz and its constructivist followers [19].

The semantic gap is "the lack of coincidence between the information that one can extract from the visual data and the interpretation that the same data have for a user in a given situation" [3]. This problem has received an enormous amount of attention in the CBIR literature (see, e.g., [4-6]) and is not the primary focus of the paper.

Despite the large number of CBIR prototypes developed over the past 15 years (see [20] for a survey), very few have experienced widespread success or become popular commercial products. One of the most successful CBIR solutions to date, perception-based image retrieval (PBIR) [21], is also among the first CBIR solutions to recognize the need to address the problem from a perceptual perspective and it does so using a psychophysical—as opposed to biologicalapproach.

We claim that the CBIR problem cannot be solved in a general way, but rather expect that specialized CBIR solutions will emerge, each of which focused on certain types of image repositories, users' needs, and query paradigms. Some of these will rely on keywords, which may be annotated in a semiautomatic fashion, some will benefit from the use of clusters and/or categories to group images according to visual or semantic similarity, respectively, and a true image retrieval solution should attempt to incorporate as many of those modules as possible. Along these lines, Figure 2 shows how the work reported in this paper (indicated by the blocks contained within the L-shaped gray area) fits in a bigger image annotation and retrieval system in which intelligent semiautomatic annotation [22] and classical query-by-visualcontent [23] capabilities are also available to the end user.

The proposed model is applicable to image retrieval scenarios where one or few ROIs are present in each image, for example, semantically relevant objects against a background or salient by design objects (such as road signs, tennis balls, emergency buttons, to name a few) in potentially busy scenes. Some of the image retrieval tasks that will not benefit from the work proposed in this paper-but that can nevertheless be addressed by other components of the entire image retrieval solution (Figure 2) -include the ones in which the gist of the scene is more closely related to its semantic meaning, and there is no specific object of interest (e.g., a sunshine scene). In this particular case, there is neurophysiological evidence [24] that attention is not needed and therefore the proposed model is not only unnecessary but also inadequate. In a complete CBIR solution, these cases can be handled by a different subsystem, focusing on global image properties, and not relying on a saliency map.

\subsection{Visual attention}

There are many varieties of attention, but in this paper we are interested in what is usually known as attention for perception: the selection of a subset of information for further processing by another part of the information processing system. In the particular case of visual information, this can be translated as "looking at something to see what it is" [25].

It is not possible for the HVS to process an image entirely in parallel. Instead, our brain has the ability to prioritize the order the potentially most important points are attended to when presented with in a new scene. The result is that much of the visual information our eyes sense is discarded. Despite, we are able to quickly gain remarkable insight into a scene. The rapid series of movements the eyes make are known as scanpaths [26]. This ability to prioritize our attention is not only efficient, but critical to survival.

There are two ways attention manifests itself. Bottomup attention is rapid and involuntary. In general, bottom-up 


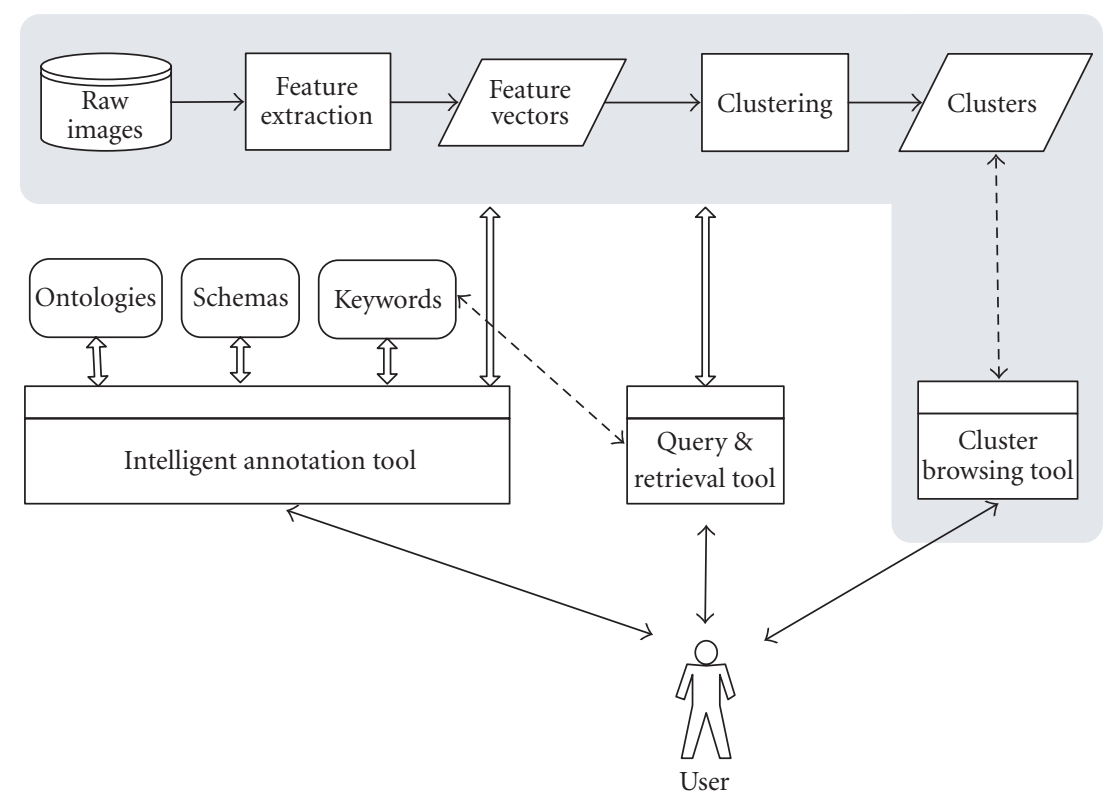

FIGURE 2: CBIR and related systems, highlighting the scope of this work.

processing is motivated by the stimulus presented [25]. Our immediate reaction to a fast movement, bright color, or shiny surface is performed subconsciously. Features of a scene that influence where our bottom-up visual attention is directed are the first to be considered by the brain and include color, movement, and orientation, among others [15]. For example, we impulsively shift our attention to a flashing light. Complementing this is attention that occurs later, controlled by top-down knowledge-what we have learned and can recall. Top-down processing is initiated by memories and past experience [25]. Looking for a specific letter on a keyboard or the face of a friend in a crowd are tasks that rely on learned, top-down knowledge.

Both bottom-up and top-down factors contribute to how we choose to focus our attention. However, the extent of their interaction is still unclear. Unlike attention that is influenced by top-down knowledge, bottom-up attention is a consistent, nearly mechanical (but purely biological) process. In the absence of top-down knowledge, a bright red stop sign will instinctively appear to be more salient than a flat, gray road. Computational modeling of visual attention (Section 2.3) has made the most progress interpreting bottom-up factors that influence attention whereas the integration of topdown knowledge into these models remain an open problem. Because of their importance, emphasized by the fact that bottom-up components of a scene influence our attention before top-down knowledge does [27] and that they can hardly be overridden by top-down goals, the proposed work focuses on the bottom-up influences on attention.

\subsubsection{Attention and similarity}

Retrieval by similarity is a central concept in CBIR systems. Similarity is based on comparisons between several images.
One of the biggest challenges in CBIR is that the user seeks semantic similarity but the CBIR system can only satisfy similarity based on physical features [3].

The notion of similarity varies depending on whether attentional resources have been allocated while looking at the image. Santini and Jain [28] distinguish preattentive similarity from attentive similarity: attentive similarity is determined after stimuli have been interpreted and classified, while preattentive similarity is determined without attempting to interpret the stimuli. They postulate that attentive similarity is limited to the recognition process while preattentive similarity is derived from image features [28].

Their work anticipated that preattentive (bottom-up) similarity would play an important role in general-purpose image databases before computational models of (bottomup) visual attention such as the ones described in Section 2.3 were available. For specialized, restricted databases, on the other hand, the use of attentive similarity could still be considered adequate, because it would be equivalent to solving a more constrained recognition problem.

\subsubsection{Attention, perception, and context}

Perception is sensory processing [25]. In terms of the visual system, perception occurs after the energy (light) that bombards the rods and cones in the eyes is encoded and sent to specialized areas of the brain. Perceptual information is used throughout to make important judgements about the safety of a scene, to identify an object, or to coordinate physical movements.

"Although the perceptual systems encode the environment around us, attention may be necessary for binding together the individual perceptual properties of an object such 
as its color, shape and location, and for selecting aspects of the environment for perceptual processes to act on" [25].

In a limited variety of tasks, such as determining the gist of a scene, perception can occur without attention [24]. However, for most other cases, attention is a critical first step in the process of perception.

Perception is not exclusively based on what we see. What we perceive is also a direct result of our knowledge and what we expect to see [30]. Many research studies have shown that the perception of a scene or the recognition of its components is strongly influenced by context information, such as recent stimuli (priming) [31] and the expected position of an object within a scene [32].

Specialized CBIR systems, by their nature, have a sense of context in that the scope is limited. However, this is certainly short of the ability to narrow the possible interpretations of an image by dynamically choosing a context. The function of nonspecialized CBIR systems may be loosely equated to the gist of a scene task. The addition of information derived from visual attention models to the CBIR scenario may signal the beginning of a new array of opportunities to incorporate context information into CBIR systems in a more realistic way.

\subsection{Biologically inspired computational models of visual attention and applications}

Several computational models of visual attention have been proposed, and they are briefly described in [33]. However, for the purpose of this paper, the two most relevant models are those proposed by Itti et al. [15] and Stentiford [16]. They are described in more detail in the following sections.

\subsubsection{The Itti-Koch model of visual attention}

The Itti-Koch model of visual attention considers the task of attentional selection from a purely bottom-up perspective, although recent efforts have been made to incorporate topdown impulses [15]. The model generates a map of the most salient points in an image, which will be henceforth referred to as long-range saliency map, or simply saliency map. Color, intensity, orientation, motion, and other features may be included as features.

The saliency map produced by the model can be used in several ways. In the work presented in this paper, we use the most salient points as cues for identifying ROIs. In a related work, Rutishauser et al. [34] apply the Itti-Koch model by extracting a region around the most salient patch of an image using region-growing techniques. Key points extracted from the detected object are used for object recognition. Repeating this process after the inhibition of return has taken place enables the recognition of multiple objects in a single image. However, this technique limits the relative object size (ROS)_-defined as the ratio of pixels belonging to the object and total number of pixels in the image - to a maximum of $5 \%$ [34].

The model has also been used in the context of object recognition [35]. Navalpakkam and Itti have begun to extend

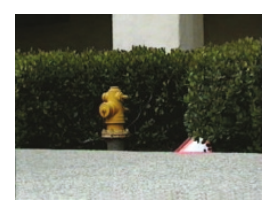

(a)

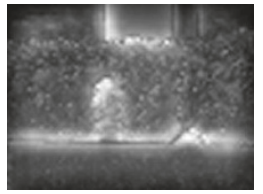

(b)

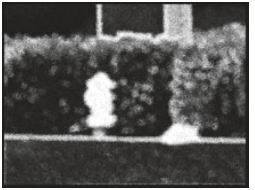

(c)
FIgure 3: Comparison between Itti-Koch and Stentiford models of visual attention: (a) original image (from http://ilab.usc .edu/imgdbs/ [29]); (b) Itti-Koch saliency map; (c) Stentiford visual attention map.

the Itti-Koch model to incorporate top-down knowledge by considering the features of a target object [36]. These features are used to bias the saliency map. For instance, if one wants to find a red object in a scene, the saliency map will be biased to consider red more than other features.

The ability of the Itti-Koch saliency model to actually predict human attention and gaze behavior has been analyzed elsewhere [37-40] and is not free of criticism. It is easy to find cases where the Itti-Koch model does not produce results that are consistent with actual fixations. The work of Henderson et al. documents one such instance where the saliency map (and computational models of visual attention in general) do not share much congruence with the eye saccades of humans [41]. However, this work adds the constraint that the visual task being measured is active search, not free viewing. The Itti-Koch model was not initially designed to include the top-down component that active search and similar tasks require.

\subsubsection{The Stentiford model of visual attention}

The model of visual attention proposed by Stentiford [16]henceforth referred to as the Stentiford model of visual attention-is also a biologically inspired approach to CBIR tasks [16]. It functions by suppressing areas of the image with patterns that are repeated elsewhere. As a result flat surfaces and textures are suppressed while unique objects are given prominence. Regions are marked as high interest if they possess features not frequently present elsewhere in the image. The result is a visual attention map that is similar in function to the saliency map generated by Itti-Koch.

The visual attention map generated by Stentiford tends to identify larger and smoother salient regions of an image, as opposed to the more focused peaks in Itti-Koch's saliency map, as illustrated in Figure 3. Thus we apply the Stentiford's visual attention map to the segmentation, not detection, of salient regions. This process is explained in more detail in Section 3.3.2. Unfortunately, the tendency of the Stentiford model to mark large regions can lead to poor results if these regions are not salient. Itti's model is much better in this regard. By identifying the unique strengths and weaknesses of each model we were able to construct our new method for extracting regions of interest. 


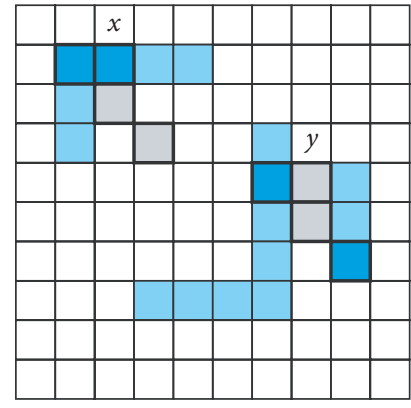

Figure 4: Matching neighborhoods $x$ and $y$ (adapted from [42]).

Figure 4 shows an example of how the Stentiford model matches random neighborhoods of pixels. In this model, digital images are represented as a set of pixels, arranged in a rectangular grid. Each pixel is assigned a visual attention (VA) score. This process starts by creating a random pattern of pixels to be sampled in the vicinity of the original pixel. This neighborhood is compared to a different, randomly selected neighborhood in the image. The degree of mismatch between the neighborhoods forms the basis for the VA score and the process continues. If the neighborhoods are identical, the VA score of a pixel will not change. As a result, the highest scoring regions are those with the smallest degree of similarity to the rest of the image. The reader is referred to [42] for a more detailed explanation.

\subsection{Related work}

The use of computational models of visual attention in CBIR-like applications has recently started and there are not too many examples of related work in the literature. In this section we briefly review three of them, which appear to be most closely related to the solution proposed in this paper.

In [43], Boccignone et al. investigate how image retrieval tasks can be made more effective by incorporating temporal information about the saccadic eye movements that a user would have followed when viewing the image, effectively bringing Ballard's animate vision paradigm [44] to the context of CBIR. They also use Itti-Koch's model to compute preattentive features which are then used to encode an image's visual contents in the form of a spatiotemporal feature vector (or "signature") known as information path (IP). Similarity between images is then evaluated on a 5000image database using the authors' IP matching algorithms. The main similarities between their work and the approach proposed in this paper are the use of Itti-Koch's model to implement (part of) the early vision stage and the application domain (CBIR). The main differences lie in the fact that our work, at this stage, relies on the long-range saliency map provided by Itti-Koch's model and does not take the temporal aspects of the scanpaths explicitly into account.

Stentiford and his colleagues have been studying the application of visual attention to image retrieval tasks. While we incorporate a part of the group's work, the Stentiford model of visual attention, into our new architecture, it is meaningful to note related applications of this model. Bamidele and Stentiford use the model to organize a large database of images into clusters [45]. This differs from our work in that no salient ROIs are extracted.

Machrouh and Tarroux have proposed using attention for interactive image exploration [46]. Their model uses past knowledge to modulate the saliency map to aid in object recognition. In some ways it is similar to the method proposed in this work, but it has key differences. Machrouh and Tarroux simulate long-term memory to implement a topdown component, our model is purely bottom-up. Additionally, their implementation requires user interaction while ours is unsupervised. The example provided by Machroux and Tarroux presents the task of face detection and detection of similar regions within a single image. This work is not concerned with intra-image similarity, but rather with inter-image relationships.

\section{THE PROPOSED MODEL}

This section presents an overview of the proposed model and explains its main components in detail.

\subsection{Overview}

We present a biologically-plausible model that extracts ROIs using saliency-based visual attention models, which are then used for the image clustering process. The proposed solution offers a promising alternative to overcoming some of the limitations of current CBIR and OBIR systems.

Our architecture incorporates a model of visual attention to compute the salient regions of an image. Regions of interest are extracted depending on their saliency. Our first cue to potential ROIs are salient peaks in the Itti-Koch saliency map. If these peaks overlap with salient regions in Stentiford's model, we proceed to extract ROIs around that point. Images are then clustered together based on the features extracted from these regions. The result is a group of images based not on their global characteristics (such as a blue sky), but rather on their salient regions. When a user is quickly viewing scenes or images the salient regions are those that stand out more quickly. Additionally, the background of an image quite often dominates the feature extraction component of many CBIR systems leading to unsatisfying results for the user.

The proposed work is based on bottom-up influences of attention and, therefore, purely unsupervised. One of the advantages of relying exclusively on bottom-up information is that bottom-up components of a scene influence our attention before top-down knowledge does [27]. Moreover, attention leads us to the relevant regions of an image and allows us to handle multiple ROIs within a scene without relying on classical segmentation approaches. When we are presented with an image of which we have no prior knowledge about and are given no instruction as to what to look for, our attention flows from salient point to point, where saliency is calculated based on only bottom-up influences. 
There are many applications of this knowledge in a variety of diverse fields. In developing user interfaces we may desire the most important functions to more easily attract our attention. For example, in cars the button to activate the hazard lights is red to distinguish itself from less critical buttons. Similarly, when we are driving through a crowded city it is important for warning signs to be the first thing we direct our attention to. Attention has also been used to compress images by enabling the automated selection of a region of interest [47].

Recent work has also shown that the performance of object recognition solutions increases when preceded by computational models of visual attention that guide the recognition system to the potentially most relevant objects within a scene [34]. We apply the same methodology to the problem of CBIR, keeping in mind the differences between the object recognition and the similarity-based retrieval tasks, namely [7], the degree of interactivity, the different relative importance of recall and precision, the broader application domains and corresponding semantic ranges, and the application-dependent semantic knowledge associated with the extracted objects (regions). In spite of these differences we believe that attention can improve image retrieval in a comparable way that it has been shown to improve the performance of object recognition solutions [34]. Since CBIR is much less strict than object recognition in terms of the quality of the object segmentation results, we settle for ROIs instead of perfectly segmented objects.

\subsection{Key aspects}

The following are the key aspects of our model.

\section{Biologically plausible}

Our model satisfies biological plausibility by combining Itti and Koch's and Stentiford's biologically inspired models of visual attention with the clustering of results, whichaccording to Draper et al. [48] —is also a biologically plausible task.

\section{Unsupervised and content-based}

It is important that our model is able to function entirely unsupervised. Groupings are made solely based on the content of the given image. Our model is able to function without the intervention of a user, producing clusters of related images at its output. These clusters can then be browsed by the user, exported to other applications, or even annotated (although this is currently not implemented).

\section{Bottom-up}

We limit our model to incorporating only bottom-up knowledge. To date, despite advances, true top-down knowledge has not been successfully incorporated into models of visual attention. Itti and Koch's work as well as derivative research has shown that promising results can still be obtained despite the lack of top-down knowledge in situations where bottom-

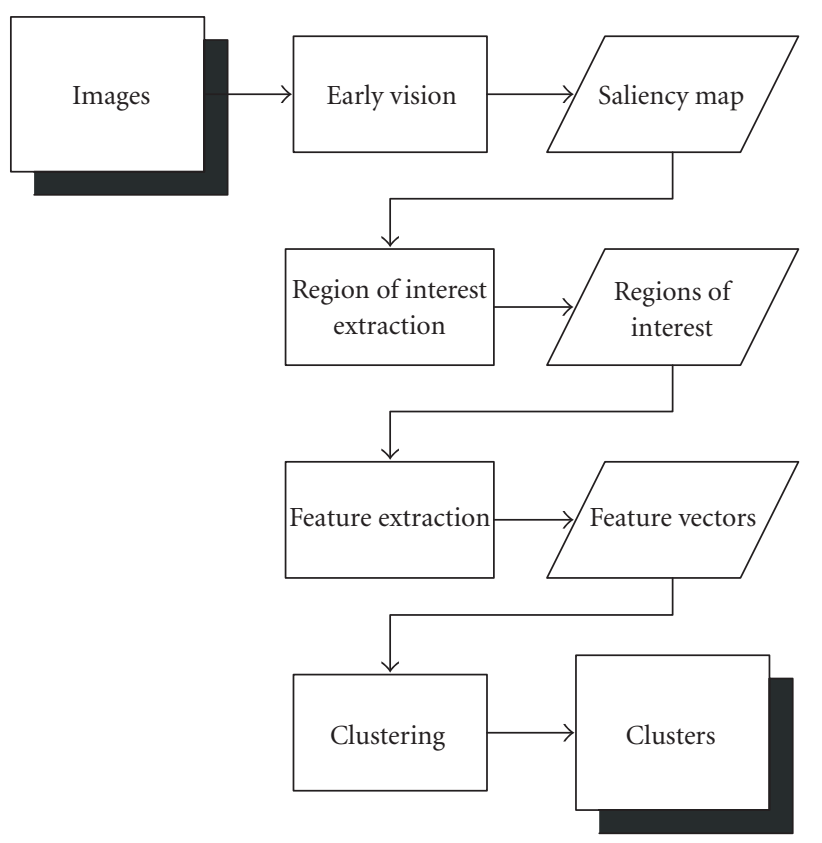

FIGURE 5: The proposed model.

up factors are enough to determine the salient region of an image [49].

\section{Modular}

While we rely on the Itti-Koch model of visual attention, our model allows for a variety of other models of visual attention to be used in its place. Similarly, the choice of feature extraction techniques and descriptors as well as clustering algorithms can also be varied. This allows a good degree of flexibility and finetuning (or customization) based on results of experiments, such as the ones described in Section 4. Additionally, our modular design means that our model is completely independent of the query, retrieval, and annotation stages of a complete CBIR solution (such as the one shown in Figure 2).

\subsection{Components}

Our model consists of the following four stages (Figure 5): early vision (visual attention), region of interest extraction, feature extraction, and clustering. The current prototype has been implemented in MATLAB and uses some of its built-in functionality, as it will be occasionally mentioned along this section.

\subsubsection{Early vision}

The first stage models early vision-specifically, what our visual attention system is able to perceive in the first few milliseconds. The purpose of this state is to indicate what the most salient areas of an image are. The input to this stage is a source image. The output is the saliency map which is based on differences in color, intensity, and orientation. We 
use the Itti-Koch model of visual attention as a proven, effective method of generating the saliency map. It has been successfully tested in a variety of applications [50]. Saliency maps were computed using a Java implementation of the Itti-Koch model of visual attention [51]. The visual attention maps proposed by Stentiford were generated by our own MATLAB implementation of the methods described in [16].

The proposed model is not domain-specific and does not impose limits on the variety of images that it applies to, provided that there is at least one semantically meaningful ROI within the image. The process of generating a saliency map and selecting the most salient ROIs reduces the impact of distractors. As noted earlier, the recognition of multiple objects cannot be done without attentional selection [34].

\subsubsection{Region of interest extraction}

The second stage of our model generates ROIs that correspond to the most salient areas of the image. It is inspired by the approach used by Rutishauser et al. [34]. Our model appreciates not only the magnitude of the peaks in the saliency map, but the size of the resulting salient regions as well. The extracted ROIs reflect the areas of the image we are likely to attend to first. Only these regions are considered for the next step, feature extraction.

The algorithm for extracting one or more regions of interest from an input image described in this paper combines the saliency map produced by the Itti-Koch model with the segmentation results of Stentiford's algorithm in such a way as to leverage the strengths of either approach without suffering from their shortcomings. More specifically, two of the major strengths of the Itti-Koch model-the ability to take into account color, orientation, and intensity to detect salient spots (whereas Stentiford's is based on color and shape only) and the fact that it is more discriminative among potentially salient regions than Stentiford's - are combined with two of the best characteristics of Stentiford's approach-the ability to detect entire salient regions (as opposed to Itti-Koch's peaks in the saliency map) and handle regions of interest larger than the 5\% ROS limit mentioned in [34].

Figure 6 shows a general view of the whole ROI extraction algorithm, using as input example the image $I$ containing a road marker and a sign (therefore, two ROIs). The basic idea is to use the saliency map produced by the Itti-Koch model to start a controlled region growing of the potential ROIs, limiting their growth to the boundaries established by Stentiford's results and/or a predefined maximum ROS. The first step is to extract the saliency $(S)$ and VA $(V)$ maps from the input image $(I)$. Both maps were explained in Sections 2.3.1 and 2.3.2, respectively. Note that while the saliency map returns small highly salient regions (peaks) over the ROIs, the VA map returns high VA score pixels for the entire ROIs, suggesting that a combination of $S$ and $V$ could be used in a segmentation process. In Figure 6, the image processing box (IPB-S) block takes $S$ as input and returns a binary image $S_{p}$ containing small blobs that are related to the most salient regions of the image. The IPB-V block takes $V$ as input and returns a binary image $V_{p}$, containing large areas with high VA scores, instead of blobs. Images $S_{p}$ and $V_{p}$ are presented to the mask generation block, that compares them and uses the matching regions as cues for selection of the ROIs into $V_{p}$. The result is a near perfect segmentation of the ROIs present in the example input image $I$.

Figure 7 presents additional details about the operations performed by the IPB-S, IPB-V and mask generation blocks.

The IPB-S block performs the following operations.

(i) Thresholding: converts a grayscale image $f(x, y)$ into a black-and-white (binary) equivalent $g(x, y)$ according to (1), where $T$ is a hard threshold in the $[0, \ldots, 255]$ range, valid for the entire image. This is accomplished by using the "im $2 b w()$ " function in MATLAB,

$$
g(x, y)= \begin{cases}1 & \text { if } f(x, y)>T \\ 0 & \text { if } f(x, y) \leq T\end{cases}
$$

(ii) Remove spurious pixels: removes undesired pixels from the resulting binarized image. This is implemented using a binary morphological operator available in the "bwmorph()" function (with the spur parameter) in MATLAB.

(iii) Remove isolated pixels: removes any remaining white pixels surrounded by eight black neighbors. This is implemented using a binary morphological operator available in the "bwmorph()" function (with the clean parameter) in MATLAB.

The IPB-V block performs thresholding (as explained above) followed by the two operations below.

(i) Morphological closing: fills small gaps within the white regions. This is implemented using a binary morphological operator, described in (2), where $\ominus$ denotes morphological erosion and $\oplus$ represents morphological dilation with a structuring element. This is accomplished by using the "imclose()" function in MATLAB,

$$
A \circ B=(A \ominus B) \oplus B .
$$

(ii) Region filling: flood-fills enclosed black regions of any size with white pixels, starting from specified points. This is implemented using a binary morphological operator available in the "imfill()" function (with the holes parameter) in MATLAB.

The mask generation block performs (self-explanatory) logical AND and OR operations, morphological closing, and region filling (as described above) plus the following steps.

(i) Find centroids: shrinks each connected region until only a pixel is left. This is accomplished by using the "bwmorph()" function (with the shrink parameter) in MATLAB.

(ii) Square relative object size (ROS): draws squares of fixed size (limited to $5 \%$ of the total image size) around each centroid.

(iii) $\mathrm{CP}$ : combines each centroid image $(C)$ with a partial $(P)$ image in order to decide which ROIs to keep and which to discard. 


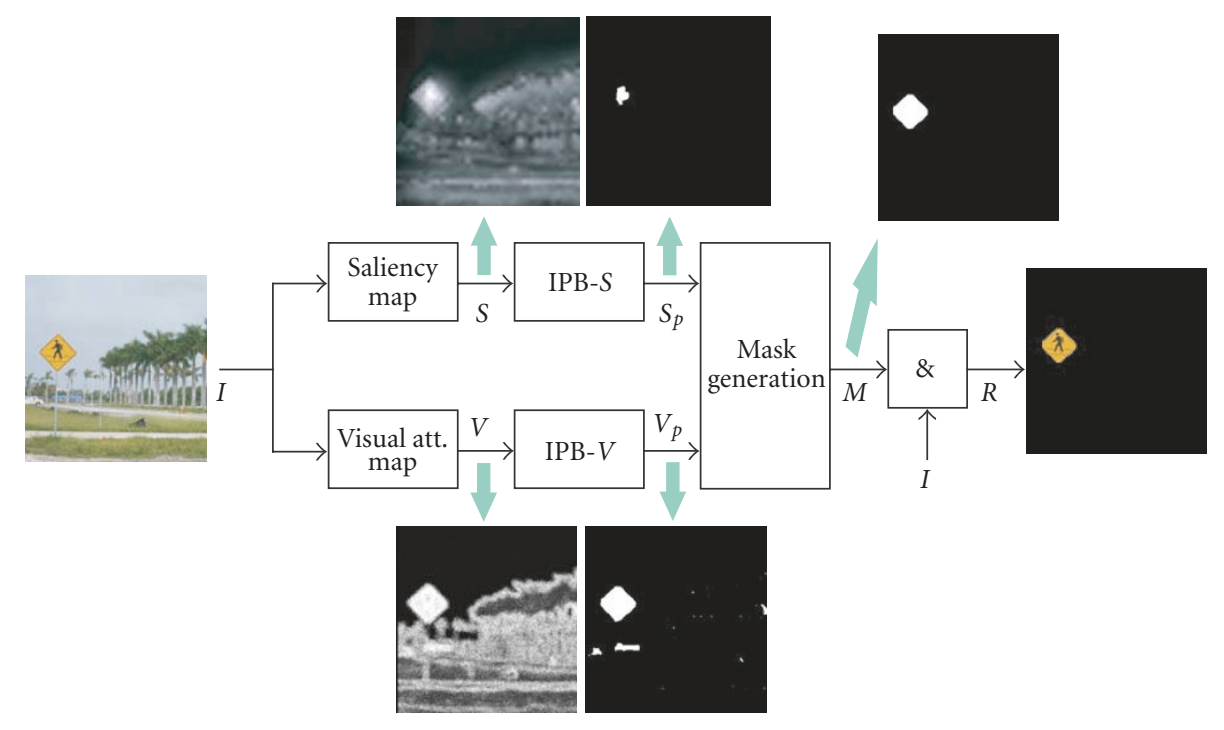

FIgURE 6: The ROI extraction algorithm: general block diagram and example results.

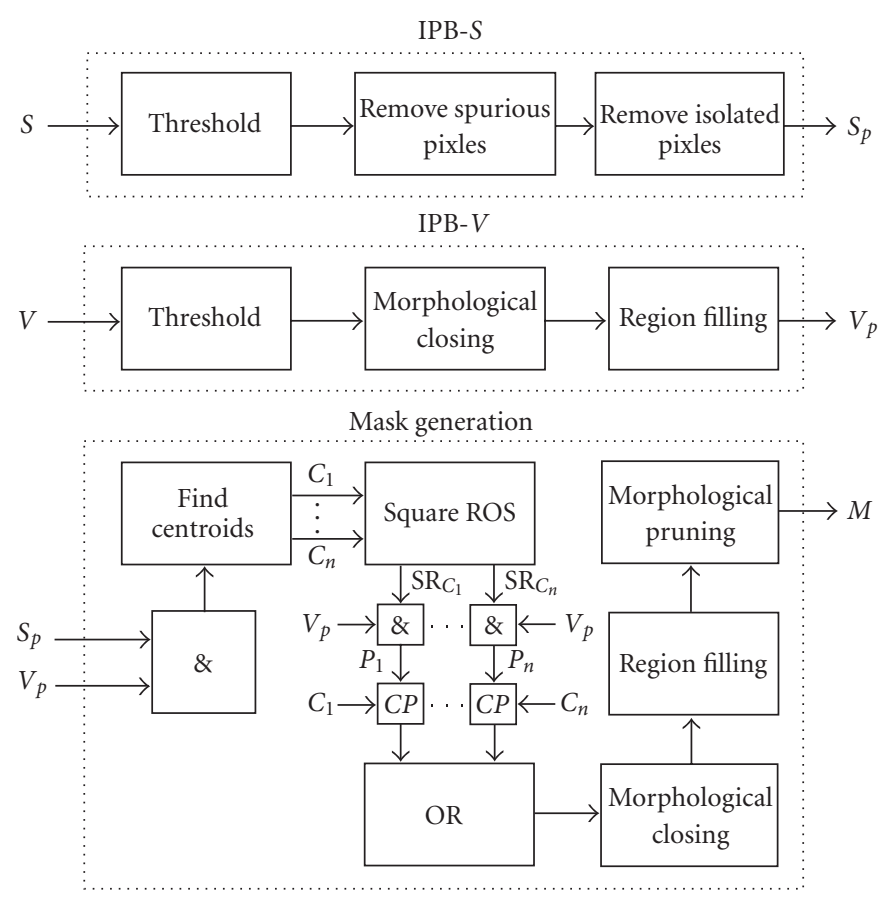

FIGURE 7: The ROI extraction algorithm: detailed block diagram.

(iv) Morphological pruning: performs a morphological opening and keeps only the largest remaining connected component, thereby eliminating smaller (undesired) branches.

The ideal result of applying our method is an image that contains the most prominent objects in a scene, discards what is not salient, handles relatively large objects, and takes into account salient regions whose saliency is due to properties other than color and shape. Figure 8 shows additional results for two different test images: the image on the left contains two reasonably large objects of interest (a traffic sign and a telephone) that are segmented successfully despite the fact that one of them resulted from prominent, but unconnected, peaks in the Itti-Koch saliency map. The image on the right-hand side of Figure 8 shows a case where Stentiford's algorithm would not perceive the tilted rectangle as more salient than any other, but-thanks to Itti-Koch model's reliance on orientation in addition to color and intensity-our algorithm segments it as the only salient region in the image. 


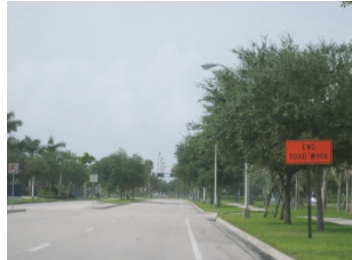

(a)

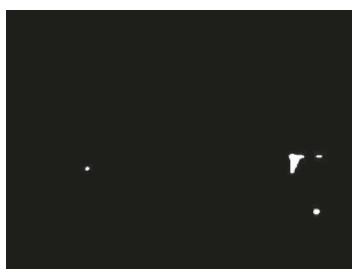

(c)

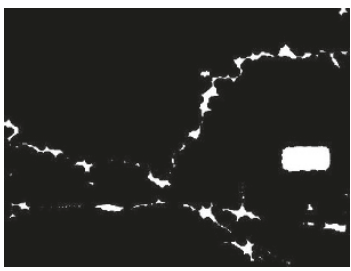

(e)

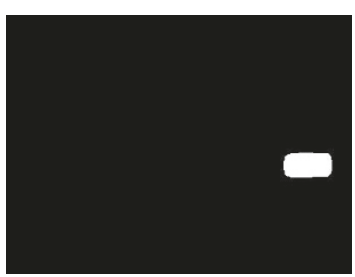

(g)

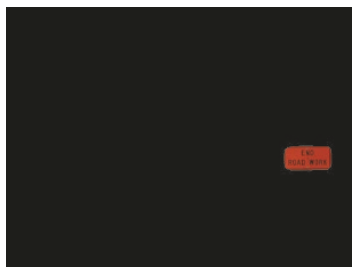

(i)

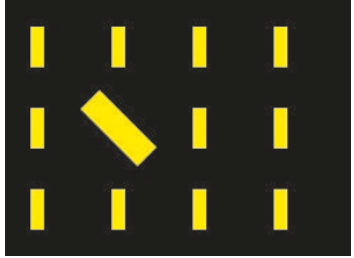

(b)

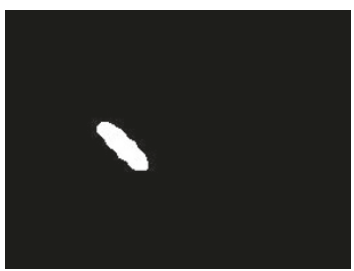

(d)

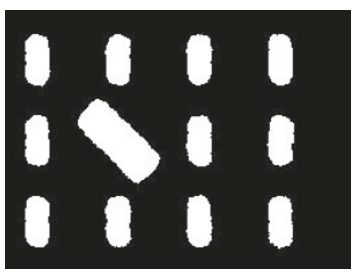

(f)

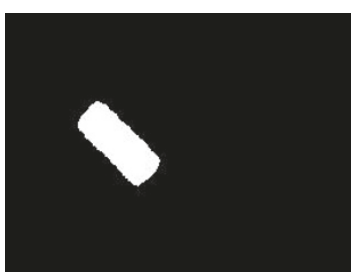

(h)

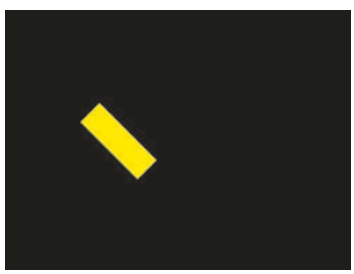

(j)
Figure 8: Examples of region of interest extraction. From top to bottom: original image (I), processed saliency map (Sp), processed Stentiford's VA map (Vp), mask (M), and final image, containing the extracted ROIs (R).

\subsubsection{Feature extraction}

The proposed system allows using any combination of feature extraction algorithms commonly used in CBIR, for example, color histograms, color correlograms, Tamura texture descriptors, Fourier shape descriptors, and so forth (see [52] for a brief comparative analysis), applied on a region-byregion basis. Each independent ROI has its own feature vec- tor. An image may be associated with several different feature vectors.

The current prototype implements two color-based feature extraction algorithms and descriptors, a 216-bin RGB color histogram and a 256-cell quantized HMMD (MPEG7-compatible) descriptor. The latter is expected to produce better results than the former, because of the chosen color space (which is closer to a perceptually uniform color space than the RGB counterpart) and due to the nonuniform subspace quantization that it undergoes.

\subsubsection{Clustering}

The final stage of our model groups the feature vectors together using a general-purpose clustering algorithm. Just as an image may have several ROIs and several feature vectors it may also be clustered in several different, entirely independent, groups. This is an important distinction between our model and other cluster-based approaches, which often limit an image to one cluster membership entry. The flexibility of having several ROIs allows us to cluster images based on the regions (objects) we are more likely to perceive rather than only global information.

Recently, Chen et al. [53] demonstrated that clustering and ranking of relevant results is a viable alternative to the usual approach of presenting the results in a ranked list format. The results of their experiments demonstrated that their approach provides clues that are semantically more relevant to a CBIR user than those provided by the existing systems that make use of similar measurement techniques. Their results also motivated the cluster-based approach taken in our work.

Figure 9 shows the results of clustering 18 images containing five ROIs with possible semantic meaning, namely: mini-basketball, tennis ball, blue plate, red newspaper stand, and yellow road sign. It can be seen that the proposed solution does an excellent job grouping together all occurrences of similar ROIs into the appropriate clusters. This simple example captures an essential aspect of the proposed solution: the ability to group together similar ROIs in spite of large differences in the background.

\section{EXPERIMENTS AND RESULTS}

This section contains representative results from our experiments and discusses the performance of the proposed approach on a representative dataset.

\subsection{Methodology}

The composition of the image database is of paramount importance to the meaningful evaluation of any CBIR system. The images must be of the appropriate context so that the results are relevant. In the case of this work it was necessary to have a database containing images with semantically well-defined ROIs (regions that are salient by design). Photographs of scenes with a combination of naturally occurring 


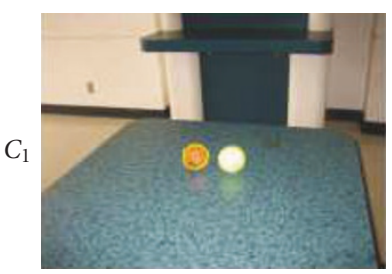

(a)

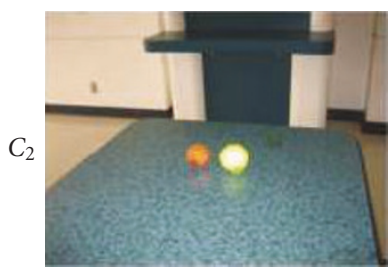

(e)

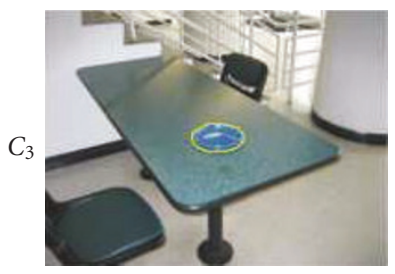

(i)

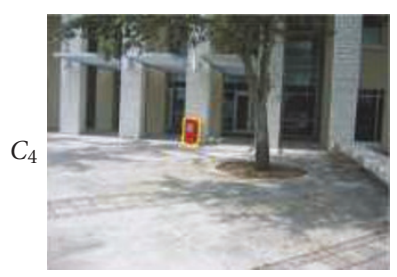

(m)

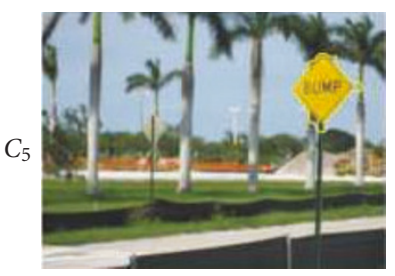

(q)

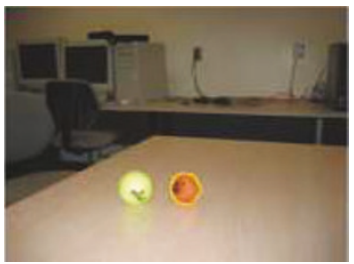

(b)

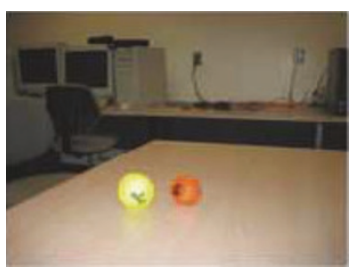

(f)

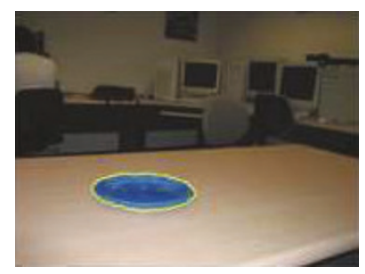

(j)

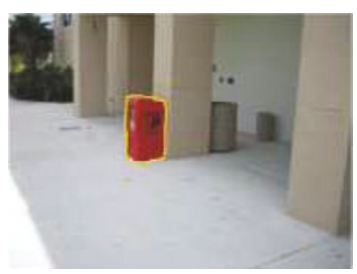

(n)

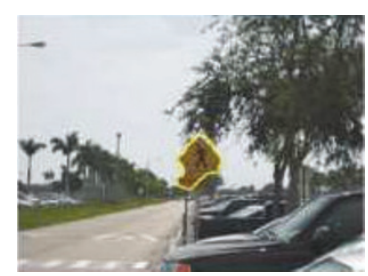

(r)

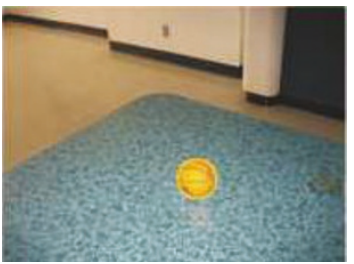

(c)

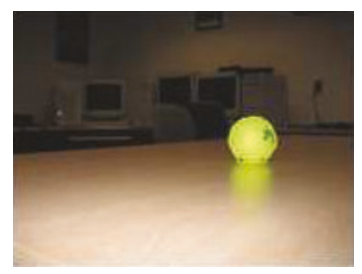

(g)

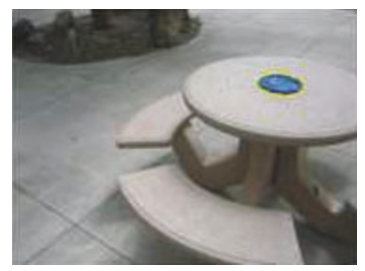

(k)

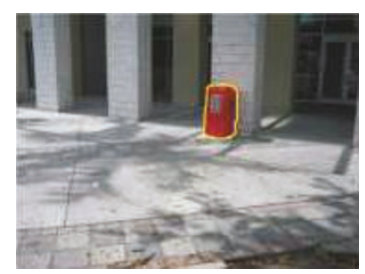

(o)

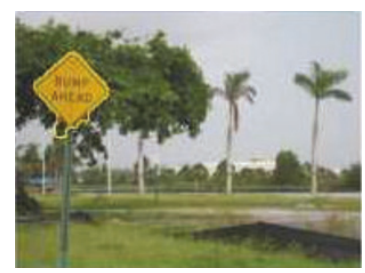

(s)

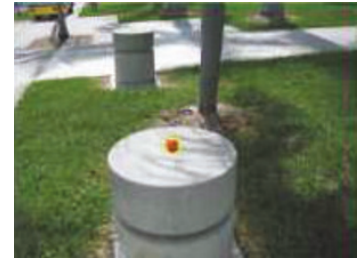

(d)

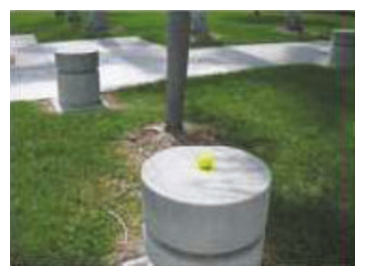

(h)

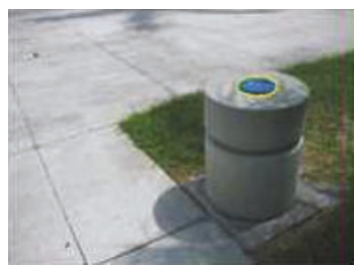

(1)

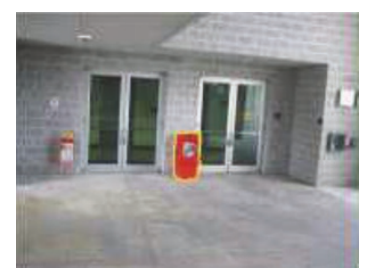

(p)

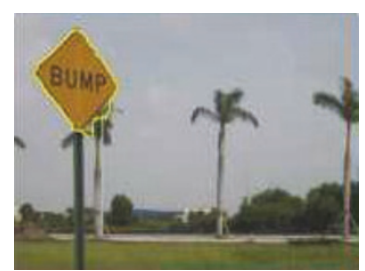

(t)

FIgURE 9: Examples of clustering based on ROIs for a small dataset. The extracted ROIs are outlined.

and artificial objects are a natural choice. Our computational model underwent preliminary assessment using a subset of images from the STIMautobahn, STIMCoke, and STIMTriangle archives available at the iLab image database repository (http://ilab.usc.edu/imgdbs/) [29]. We selected a total of 110 images, divided as follows: 41 images from the STIMau- tobahn database (a variety of road signs), 41 images from the STIMCoke database (red soda cans in many different sizes, positions and backgrounds), and 28 images from the STIMTriangle database (emergency triangles in many different relative sizes, positions, and backgrounds). The resulting database provided a diverse range of images with an 

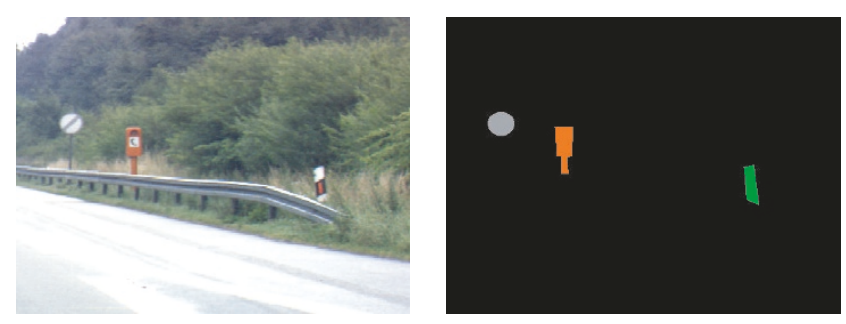

FIgure 10: The ground truth ROIs for a sample image. The image on the left can be found at http://ilab.usc.edu/imgdbs/ [29].

appropriate balance between easy, moderate, and difficultto-isolate ROIs.

An initial manual analysis of the selected 110 images was done to establish the ground truth ROIs. In total, 174 regions were divided between 22 clusters. In the ground truth, for example, all red soda cans belong to one cluster, while all orange signs belong to another. The ground truth was agreed upon by three people familiar with the images and is not ambiguous. Identified ROIs are shown for one of the images included in the database in Figure 10.

For each image the corresponding saliency map was extracted and used to compute the relevant ROIs using the algorithm described in Section 3.3.2. Each ROI was encoded using either a 27-bin RGB color histogram or a 32-cell quantized HMMD color descriptor (both have been described in Section 3.3.3) as the feature vector. The resulting feature vectors were clustered using the classic $K$-means clustering algorithm [54]. In-depth analysis of these results is presented in Section 4.2. Further experiments tested a variety of different clustering algorithms. Their results are also qualitatively compared.

The chosen feature extraction and clustering algorithms are simple and widely accepted methods-baseline case for both stages. While the use of more sophisticated feature extraction and clustering algorithms provides more possibilities for improving the performance of the presented system, they are beyond the scope of this paper. The ability to provide meaningful results with simple modules for clustering and feature extraction provides encouragement for the potential of future work to improve this model.

\subsection{Results}

The following sections report the results of two distinct evaluation stages: ROI extraction and clustering.

\subsubsection{ROl extraction}

For ROI extraction a receiver operating characteristic (ROC) curve was generated to evaluate the ideal key parameter, the binarizing threshold of the saliency map. This curve is shown in Figure 11. It was generated by evaluating the number of true positives, false positives, and false negatives in the resulting images. The resulting figure plots the false alarm rate ver-

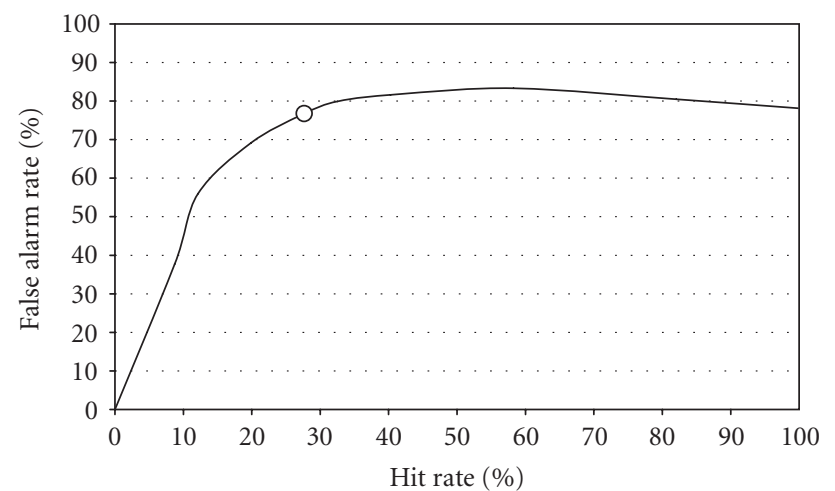

FIgURE 11: ROC curve used to evaluate the performance of the ROI extraction algorithm as a function of the threshold used to binarize the saliency map. The vertical axis represents the false alarm rate (expressed in \%), whereas the horizontal axis represents the hit rate (also expressed in \%).

sus the hit rate. ROC curves provide a visual indication of the interaction between the risk of a false positive and the reward of a true positive and facilitate the selection of a threshold.

The false alarm rate is defined as

$$
\text { false alarm rate }=\frac{\mathrm{FP}}{\max (\mathrm{FP})} .
$$

The hit rate is defined as

$$
\text { hit rate }=\frac{\mathrm{TP}}{(\mathrm{TP}+\mathrm{FN})},
$$

where TP is the number of true positives, FP is the number of false positives, and $\mathrm{FN}$ is the number of false negatives.

The varied parameter of the ROC curve is the threshold used to binarize the saliency map that results from applying the Itti-Koch model of visual attention to the source image. The threshold used directly affects the potential amount of seed points provided to the further stages of the model and has a great impact on performance. If the threshold is too high not enough seeds will be generated and valid ROIs will be missed. Conversely, a low threshold will result in too many false positives. Our experiments showed that a value of 190 yielded the most balanced results—a $27.67 \%$ false alarm rate and a $76.74 \%$ hit rate.

An alternative way to determine the best value for the threshold is to compute precision $(p)$, recall $(r)$, and F1, defined as follows:

$$
p=\frac{\mathrm{TP}}{(\mathrm{TP}+\mathrm{FP})}, \quad r=\frac{T P}{(T P+F N)}, \quad \mathrm{F} 1=\frac{2 \times p \times r}{(p+r)},
$$

where $T P$ is the number of true positives, $F P$ is the number of false positives, and $F N$ is the number of false negatives. 


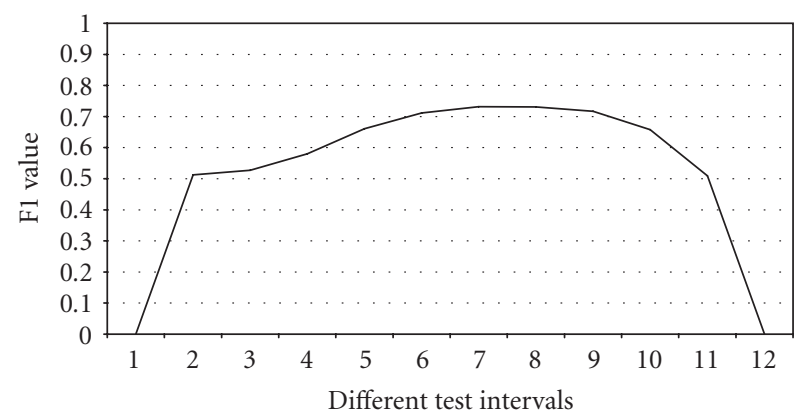

FIgURE 12: Variation of F1 as a function of the threshold used to binarize the saliency map. The vertical axis represents the F1 value, whereas the horizontal axis represents the different test intervals.

The ideal value for $p, r$, or F1 is 1 . Figure 12 shows the variation of $\mathrm{F} 1$ as a function of the threshold. Once again, the curve peaks (at about 0.73) for threshold values between 180 and 190 (intervals labeled 7 and 8 on the curve).

\subsubsection{Clustering}

Quantitative evaluation of the clustering stage was performed on raw confusion matrices obtained for each relevant case. The analysis was done from two different angles: (i) we used measures of purity and entropy (defined in (6) and (7) below) to evaluate the quality of the resulting clusters; and (ii) we adopted measures of precision, recall, and F1 to capture how well a certain semantic category was represented in the resulting clustering structure.

Given a number of categories $c$, we can define purity as

$$
p\left(C_{j}\right)=\frac{1}{\left|C_{j}\right|} \max _{k=1, \ldots, c}\left|C_{j, k}\right|
$$

while entropy can be defined as

$$
h\left(C_{j}\right)=-\frac{1}{\log c} \sum_{k=1}^{c} \frac{\left|C_{j, k}\right|}{\left|C_{j}\right|} \log \frac{\left|C_{j, k}\right|}{\left|C_{j}\right|}
$$

where $\left|C_{j}\right|$ is the size of cluster $j$, and $\left|C_{j, k}\right|$ represents the number of images in cluster $j$ that belong to category $k$.

Purity values may vary between $1 / c$ and 1 (best), whereas entropy values may vary between 0 (best) and 1 .

In the context of clustering,

$$
\begin{gathered}
p_{k}=\frac{\left|C_{j, k}\right|}{\left|C_{j}\right|}, \\
r_{k}=\frac{\left|C_{j, k}\right|}{\left|C_{k}\right|}, \\
\mathrm{F} 1_{k}=\frac{2 \times p_{k} \times r_{k}}{\left(p_{k}+r_{k}\right)},
\end{gathered}
$$

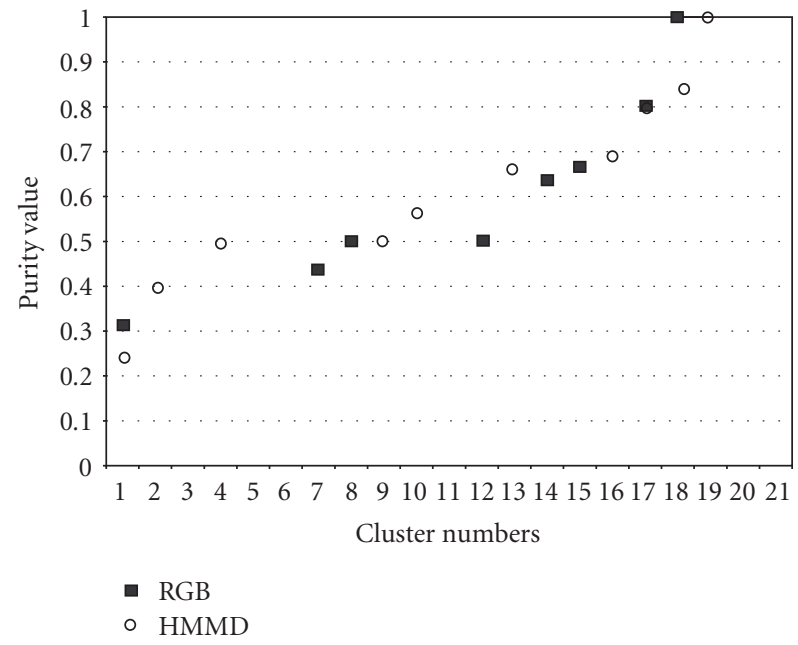

Figure 13: Measure of purity for each of the $K=21$ clusters. The vertical axis represents the purity value, whereas the horizontal axis represents the cluster numbers.

where $\left|C_{j}\right|$ is the size of cluster $j,\left|C_{j, k}\right|$ represents the number of images in cluster $j$ that belong to category $k$, and $\left|C_{k}\right|$ represents the total number of images that belong to category $k$.

The two relevant cases reported in this section used the same clustering algorithm ( $K$-Means, where $K=21$ ) but differed in the choice of feature vector (descriptor): 27-bin RGB histogram or 32-cell quantized HMMD descriptor. These two feature extraction methods were evaluated in connection with the clustering algorithms, under the rationale that the quality of resulting clusters is dependent on the quality of the input feature vectors. The value of $K=21$ here was determined independently of the number of clusters in the ground truth dataset, which was 22 .

Figure 13 shows the variation in the measure of purity for both cases, whereas Figure 14 shows the corresponding plot for measures of entropy. In both cases, the values have been sorted so that best results appear on the right-hand side of each figure. For both figures of merit, the HMMD descriptor outperforms the RGB histogram in almost all clusters.

Figure 15 shows the variation in the measure of maximum value of F1 for both cases. Once again, the HMMD descriptor outperforms the RGB histogram in almost all clusters.

We have also collected qualitative and quantitative results for other clustering algorithms, namely, fuzzy c-means, hierarchical clustering, and a combination of subtractive clustering-for seed selection-and $K$-means. The detailed quantitative comparison is beyond the scope of this paper and will be presented in a separate work. Qualitative results confirm that improved clustering algorithms can result in better clustering structures (from a human user's expectation) based upon the same feature descriptor(s) than the baseline case presented above. 


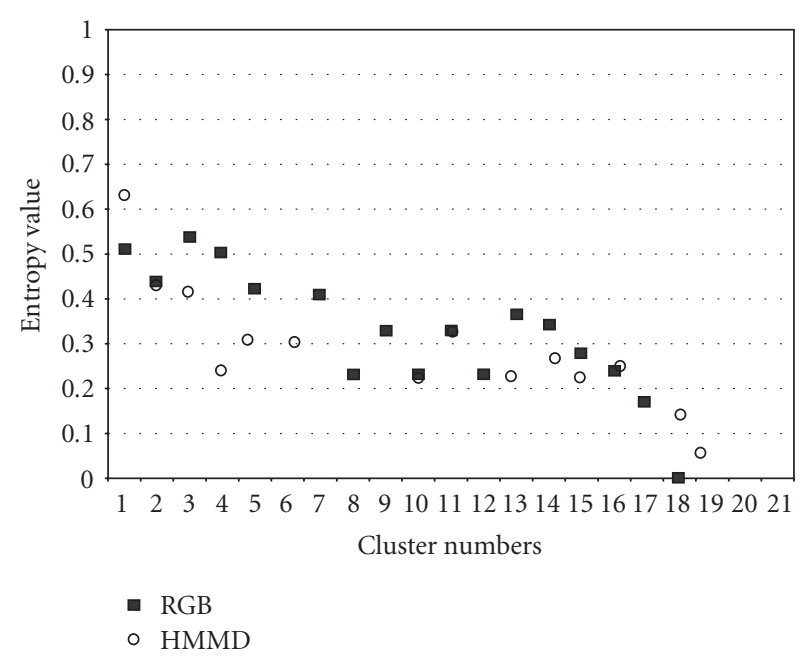

FIgure 14: Measure of entropy for each of the $K=21$ clusters. The vertical axis represents the entropy value, whereas the horizontal axis represents the cluster numbers.

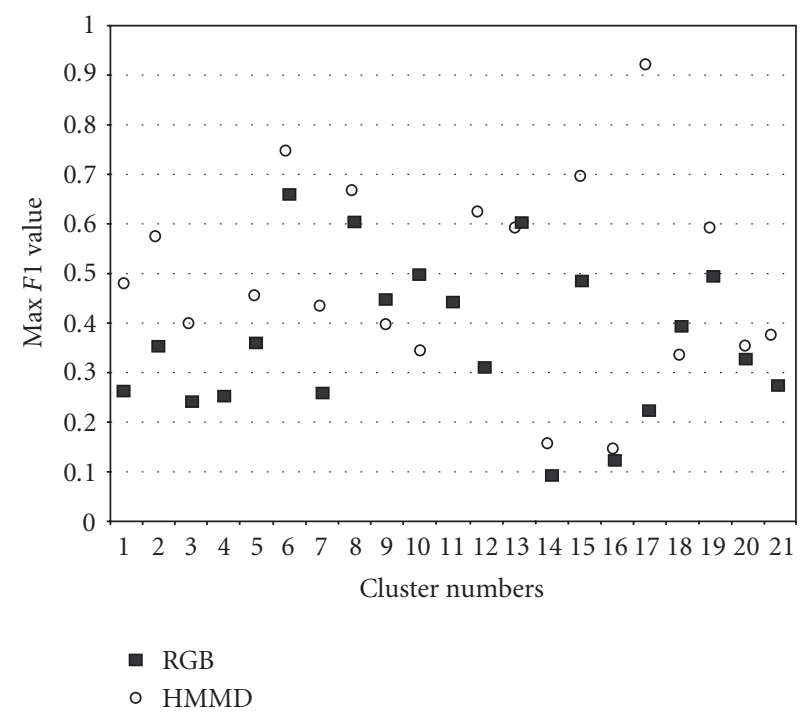

FIGURE 15: Measure of maximum value of F1 for each of the 21 semantic categories. The vertical axis represents the best (maximum) value for a certain semantic category across all clusters, whereas the horizontal axis represents the cluster numbers.

\subsection{Discussion}

Results from our experiments on a 110-image dataset containing a total of 174 ROIs and at least one ROI per image have shown that the proposed solution has performed well in most cases. The vast majority (77\% for the chosen threshold value) of meaningful ROIs are successfully extracted and eventually clustered along with other visually similar ROIs in a way that closely matches the human user's expectations.

The current ROI extraction algorithm has certain shortcomings that fall into one of the following three categories: false negatives (meaningful ROIs are not extracted), false
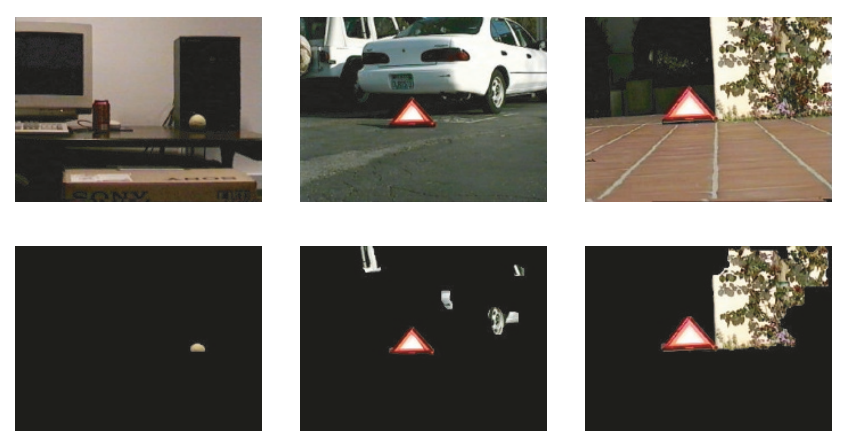

FIGURE 16: Examples of cases where the proposed ROI extraction algorithm does not work as expected. The images on the top row can be found at http://ilab.usc.edu/imgdbs/ [29].

positives (additional extraneous ROIs are extracted), and imperfect ROIs. Imperfections in the resulting ROIs can be seen in the form of incomplete, oddly shaped, and/or excessively large ROIs. Figure 16 shows three such cases. In the first one (left column), a relevant object (Coke can) is not extracted (primarily due to the poor lighting conditions of the scene). In the second case (middle column), a relatively large number of false positives appear (in addition to the only true positive in the scene, the emergency triangle). Finally, in the third case (right column), an artificially large ROI is obtained, including the object of interest (triangle), but adding many more unnecessary pixels to the ROI.

The behavior displayed by our ROC curve may initially appear to be inconsistent with the expected monotonically increasing function. Traditionally, the amount of hits will increase with more liberal criteria, at the expense of encountering more misses. However, the parameter our method uses, the threshold of the saliency map (ultimately, the number of seeds used to extract regions of interest), exhibits diminishing returns after a point. This is because low thresholds will increasingly generate more seeds and, as a result, larger regions of interest. The breaking point occurs when the ROIs get too large and start to overlap, diminishing the ability to distinguish multiple ROIs. As a result, it is not beneficial (in terms of detecting the maximum possible amount of ROIs) to continually decrease the threshold.

There are obvious dependencies among certain blocks, particularly: (i) ROI extraction and feature extraction, since a missed ROI (false negative) will never again become available to have its features extracted; (ii) feature extraction and clustering, since different descriptors will provide variations in the clustering results.

The combined tests investigating the relative impact of the chosen feature extraction algorithm on the quality of the clustering results have confirmed that the HMMD descriptor outperforms its RGB counterpart.

Interestingly enough, the feature extraction and clustering algorithms can still provide good results even in the presence of less-than-perfect results from the ROI extraction stage, as indicated in the top-most figure in cluster C5 in Figure 9. 
Our clustering experiments use the results of the ROI extraction algorithm (and subsequent feature extraction) without modification. In other words, due to the presence of false positives in the ROI extraction stage, we had to revisit the semantic categories and account for the false positives. Had we removed the false positives (which one could compare to a user-initiated action), we would have achieved much better results in the clustering stage, but would have sacrificed the unsupervised nature of our approach.

\section{CONCLUSION}

This paper presented a model for grouping images based on their salient regions. The differential of our model is that it overcomes some of the main limitations of existing object-based image retrieval solutions. It makes use of the results of a biologically inspired bottom-up model of visual attention-encoded in a saliency map-to guide the process of detecting - in a purely unsupervised manner - the most salient points within an image. These salient points are then used to extract regions of interest around them. These regions-which in many cases correspond to semantically meaningful objects - are then processed by a feature extraction module and the results are used to assign a region (and the image to which it belongs) to a cluster. Next, images containing perceptually similar objects are grouped together, regardless of the number of occurrences of an object or any distracting factors around them.

Quantitative and qualitative results of our experiments on a 110-image dataset are very encouraging and suggest that the approach should be extended and improved in ways such as described below.

Some shortcomings of the proposed solution are related to the purely bottom-up nature of the visual saliency maps upon which much of the processing is done. Future work includes, among other things, a deeper study of image retrieval users' needs to determine how they can modulate the saliency map and provide a top-down component to the current model, comparable to the work reported in [36] for target detection tasks.

Certain limitations of the proposed approach are due to its purely unsupervised nature. Since many CBIR solutions postulate the use of relevance feedback (RF) to allow user interaction, we are planning on extending the existing prototype to include an RF option (e.g., by allowing users to click on some of the false negatives and false positives obtained at the output of the ROI extraction to indicate what the algorithm missed and/or incorrectly added).

Future work also includes the extension of our system to incorporate relevance feedback at the end of the clustering stage. While clusters provide the ability to quickly pass reasonable judgment on groups of similar images, adding relevance feedback would enable the user to converge towards meaningful results with minimal interaction. We are currently considering persistent relevance feedback strategies that modify cluster membership based on the activity of multiple users across multiple sessions.
There are other areas where notable improvements could be achieved under the new model. While the feature extraction and clustering methods selected were purposefully simple, an evaluation of the performance of alternative methods in the context of this solution may lead to better overall results.

\section{ACKNOWLEDGMENT}

This research was partially sponsored by the UOL (http:// www.uol.com.br), through its UOL Bolsa Pesquisa program, no. 200503312101a and by the Office of Naval Research (ONR) under the Center for Coastline Security Technology Grant N00014-05-C-0031.

\section{REFERENCES}

[1] O. Marques and B. Furht, Content-Based Image and Video Retrieval, Kluwer Academic, Boston, Mass, USA, 2002.

[2] Y. Rui, T. S. Huang, and S.-F. Chang, "Image retrieval: current techniques, promising directions, and open issues," Journal of Visual Communication and Image Representation, vol. 10, no. 1, pp. 39-62, 1999.

[3] A. M. W. Smeulders, M. Worring, S. Santini, A. Gupta, and R. Jain, "Content-based image retrieval at the end of the early years," IEEE Transactions on Pattern Analysis and Machine Intelligence, vol. 22, no. 12, pp. 1349-1380, 2000.

[4] P. G. B. Enser and C. J. Sandom, "Towards a comprehensive survey of the semantic gap in visual image retrieval," in Proceedings of the 2nd International Conference on Image and Video Retrieval (CIVR '03), pp. 291-299, Urbana-Champaign, Ill, USA, July 2003.

[5] R. Zhao and W. I. Grosky, "Narrowing the semantic gapimproved text-based web document retrieval using visual features," IEEE Transactions on Multimedia, vol. 4, no. 2, pp. 189200, 2002.

[6] R. Zhao and W. I. Grosky, "Negotiating the semantic gap: from feature maps to semantic landscapes," Pattern Recognition, vol. 35, no. 3, pp. 593-600, 2002.

[7] C. Colombo and A. Del Bimbo, "Visible image retrieval," in Image Databases: Search and Retrieval of Digital Imagery, V. Castelli and L. D. Bergman, Eds., chapter 2, pp. 11-33, John Wiley \& Sons, New York, NY, USA, 2002.

[8] C. H. C. Leung and H. H.-S. Ip, "Benchmarking for contentbased visual information search," in Proceedings of the 4th International Conference on Advances in Visual Information Systems (VISUAL '00), pp. 442-456, Lyon, France, November 2000.

[9] H. Müller, W. Müller, and D. M. Squire, "Automated benchmarking in content-based image retrieval," in Proceedings of the IEEE International Conference on Multimedia and Expo (ICME '01), p. 290, Tokyo, Japan, August 2001.

[10] C. Carson, S. Belongie, H. Greenspan, and J. Malik, "Blobworld: image segmentation using expectation-maximization and its application to image querying," IEEE Transactions on Pattern Analysis and Machine Intelligence, vol. 24, no. 8, pp. 1026-1038, 2002.

[11] W.-Y. Ma and B. S. Manjunath, "NeTra: a toolbox for navigating large image databases," Multimedia Systems, vol. 7, no. 3, pp. 184-198, 1999. 
[12] Y. Li and L. Shapiro, "Object recognition for contentbased image retrieval," http://www.cs.washington.edu/homes/ shapiro/.

[13] D. Hoiem, R. Sukthankar, H. Schneiderman, and L. Huston, "Object-based image retrieval using the statistical structure of images," in Proceedings of the IEEE Computer Society Conference on Computer Vision and Pattern Recognition (CVPR '04), vol. 2, pp. 490-497, Washington, DC, USA, June-July 2004.

[14] Y. Tao and W. I. Grosky, "Image matching using the OBIR system with feature point histograms," in Proceedings of the 4th Working Conference on Visual Database Systems (VDB '98), pp. 192-197, L’Aquila, Italy, May 1998.

[15] L. Itti, C. Koch, and E. Niebur, "A model of saliency-based visual attention for rapid scene analysis," IEEE Transactions on Pattern Analysis and Machine Intelligence, vol. 20, no. 11, pp. 1254-1259, 1998.

[16] F. W. M. Stentiford, "An attention based similarity measure with application to content-based information retrieval," in Storage and Retrieval for Media Databases, vol. 5021 of Proceedings of SPIE, pp. 221-232, Santa Clara, Calif, USA, January 2003.

[17] R. Baeza-Yates and B. Ribeiro-Neto, Modern Information Retrieval, Addison-Wesley/ACM Press, New York, NY, USA, 1999.

[18] S.-F. Chang, J. R. Smith, M. Beigi, and A. Benitez, "Visual information retrieval from large distributed online repositories," Communications of the ACM, vol. 40, no. 12, pp. 63-71, 1997.

[19] S. Palmer, Vision Science: Photons to Phenomenology, MIT Press, Cambridge, Mass, USA, 1999.

[20] R. Veltkamp and M. Tanase, "A survey of content-based image retrieval systems," in Content-Based Image and Video Retrieval, O. Marques and B. Furht, Eds., chapter 5, pp. 47-101, Kluwer Academic, Boston, Mass, USA, 2002.

[21] E. Chang, K.-T. Cheng, W.-C. Lai, C.-T. Wu, C. Chang, and Y.L. Wu, "PBIR: perception-based image retrieval-a system that can quickly capture subjective image query concepts," in Proceedings of the 9th ACM International Conference on Multimedia, pp. 611-614, Ottawa, Canada, September 2001.

[22] O. Marques and N. Barman, "Semi-automatic semantic annotation of images using machine learning techniques," in Proceedings of the 2nd International Semantic Web Conference (ISWC '03), vol. 2870 of Lecture Notes in Computer Science, pp. 550-565, Sanibel Island, Fla, USA, October 2003.

[23] O. Marques and B. Furht, "MUSE: a content-based image search and retrieval system using relevance feedback," Multimedia Tools and Applications, vol. 17, no. 1, pp. 21-50, 2002.

[24] A. Oliva, "Gist of a scene," in Neurobiology of Attention, L. Itti, G. Rees, and J. Tsotsos, Eds., chapter 41, pp. 251-256, Academic Press, Elsevier, New York, NY, USA, 2005.

[25] E. A. Styles, Attention, Perception, and Memory: An Integrated Introduction, Taylor \& Francis Routledge, New York, NY, USA, 2005.

[26] D. Noton and L. Stark, "Scanpaths in eye movements during pattern perception," Science, vol. 171, no. 968, pp. 308-311, 1971.

[27] C. Connor, H. Egeth, and S. Yantis, "Visual attention: bottomup versus top-down," Current Biology, vol. 14, no. 19, pp. R850-R852, 2004.

[28] S. Santini and R. Jain, "The graphical specification of similarity queries," Journal of Visual Languages and Computing, vol. 7, no. 4, pp. 403-421, 1996.

[29] L. Itti and C. Koch, "Feature combination strategies for saliency-based visual attention systems," Journal of Electronic Imaging, vol. 10, no. 1, pp. 161-169, 2001.
[30] Z. W. Pylyshyn, Seeing and Visualizing: It's Not What You Think, MIT Press, Cambridge, Mass, USA, 2006.

[31] S. Palmer, "The effects of contextual scenes on the identification of objects," Memory \& Cognition, vol. 3, no. 5, pp. 519$526,1975$.

[32] I. Biederman, "Perceiving real-world scenes," Science, vol. 177, no. 43, pp. 77-80, 1972.

[33] L. Itti and C. Koch, "Computational modeling of visual attention,” Nature Reviews Neuroscience, vol. 2, no. 3, pp. 194-203, 2001.

[34] U. Rutishauser, D. Walther, C. Koch, and P. Perona, "Is bottom-up attention useful for object recognition?" in Proceedings of the IEEE Computer Society Conference on Computer Vision and Pattern Recognition (CVPR '04), vol. 2, pp. 37-44, Washington, DC, USA, June-July 2004.

[35] D. Walther, L. Itti, M. Riesenhuber, T. Poggio, and C. Koch, "Attentional selection for object recognition-a gentle way," in Proceedings of the 2nd International Workshop on Biologically Motivated Computer Vision (BMCV '02), vol. 2525 of Lecture Notes In Computer Science, pp. 472-479, Tubingen, Germany, November 2002.

[36] V. Navalpakkam and L. Itti, "Modeling the influence of task on attention," Vision Research, vol. 45, no. 2, pp. 205-231, 2005.

[37] W. Einhäuser and P. König, "Does luminance-contrast contribute to a saliency map for overt visual attention?" European Journal of Neuroscience, vol. 17, no. 5, pp. 1089-1097, 2003.

[38] D. Parkhurst, K. Law, and E. Niebur, "Modeling the role of salience in the allocation of overt visual attention," Vision Research, vol. 42, no. 1, pp. 107-123, 2002.

[39] D. Parkhurst and E. Niebur, "Texture contrast attracts overt visual attention in natural scenes," European Journal of Neuroscience, vol. 19, no. 3, pp. 783-789, 2004.

[40] R. J. Peters, A. Iyer, L. Itti, and C. Koch, "Components of bottom-up gaze allocation in natural images," Vision Research, vol. 45, no. 18, pp. 2397-2416, 2005.

[41] J. M. Henderson, J. R. Brockmole, M. S. Castelhano, and M. Mack, "Image salience versus cognitive control of eye movements in real-world scenes: evidence from visual search," in Eye Movement Research: Insights Into Mind and Brain, R. van Gompel, M. Fischer, W. Murray, and R. Hill, Eds., Elsevier, Amsterdam, The Netherlands, in press.

[42] A. Bamidele, F. W. M. Stentiford, and J. Morphett, "An attention-based approach to content-based image retrieval," BT Technology Journal, vol. 22, no. 3, pp. 151-160, 2004.

[43] G. Boccignone, A. Picariello, V. Moscato, and M. Albanese, "Image similarity based on animate vision: information path matching," in Proceedings of the 8th International Workshop on Multimedia Information Systems (MIS '02), pp. 66-75, Tempe, Ariz, USA, October 2002.

[44] D. H. Ballard, "Animate vision," Artificial Intelligence, vol. 48, no. 1 , pp. 57-86, 1991.

[45] A. Bamidele and F. W. M. Stentiford, "An attention based similarity measure used to identify image clusters," in Proceedings of the 2nd European Workshop on the Integration of Knowledge, Semantics and Digital Media Technology, London, UK, November-December 2005.

[46] J. Machrouh and P. Tarroux, "Attentional mechanisms for interactive image exploration," EURASIP Journal on Applied Signal Processing, vol. 2005, no. 14, pp. 2391-2396, 2005.

[47] A. P. Bradley and F. W. M. Stentiford, "JPEG 2000 and region of interest coding," in Proceedings of Digital Image Computing: Techniques and Applications (DICTA '02), pp. 303-308, Melbourne, Australia, January 2002. 
[48] B. Draper, K. Baek, and J. Boody, "Implementing the expert object recognition pathway," in Proceedings of the 3rd International Conference on Vision Systems (ICVS '03), Graz, Austria, April 2003.

[49] L. Itti, C. Gold, and C. Koch, "Visual attention and target detection in cluttered natural scenes," Optical Engineering, vol. 40, no. 9, pp. 1784-1793, 2001.

[50] L. Itti and C. Koch, "A saliency-based search mechanism for overt and covert shifts of visual attention," Vision Research, vol. 40, no. 10-12, pp. 1489-1506, 2000.

[51] R. Newcombe, "An interactive bottom-up visual attention toolkit in Java," http://privatewww.essex.ac.uk/ ranewc/ research/visualAttentionJava.html.

[52] W.-Y. Ma and H. J. Zhang, "Benchmarking of image features for content-based retrieval," in Proceedings of the 32nd IEEE Conference Record of the Asilomar Conference on Signals, Systems and Computers, vol. 1, pp. 253-256, Pacific Grove, Calif, USA, November 1998.

[53] Y. Chen, J. Z. Wang, and R. Krovetz, "CLUE: cluster-based retrieval of images by unsupervised learning," IEEE Transactions on Image Processing, vol. 14, no. 8, pp. 1187-1201, 2005.

[54] L. Kaufman and P. Rousseeuw, Finding Groups in Data: An Introduction to Cluster Analysis, John Wiley \& Sons, New York, NY, USA, 1990.

Oge Marques is an Assistant Professor in the Department of Computer Science and Engineering at Florida Atlantic University in Boca Raton, Florida. He received his B.S. degree in electrical engineering from Federal University of Technology Paraná (UTFPR) in Curitiba, Brazil, a Master's degree in electronic engineering from Philips International Institute of Technological Studies in Eindhoven, The Netherlands, and a Ph.D. degree in computer engineering from Florida Atlantic University. His research interests and publications have been focused on image processing, analysis, annotation, search, and retrieval; human and computer vision; video processing and analysis; and secure multimedia communications. He is a Member of ACM, IEEE, IEEE Computer Society, and the honor societies of Phi Kappa Phi and Upsilon Pi Epsilon.

Liam M. Mayron is a Ph.D. candidate with the Department of Computer Science and Engineering at Florida Atlantic University. He received his M.S. degree from the University of Florida. His research interests include content-based image retrieval, biologically inspired computing, data mining, and the Internet.

Gustavo B. Borba is a Ph.D. student with the Department of Electronics at Federal University of Technology, Paraná (UTFPR) and a Lecturer at Ensitec School of Technology, Curitiba, Brazil. He received his B.S. degree in electrical engineering and Master's degree from UTFPR. His research interests include content-based image retrieval, image processing, biologically inspired computing and embedded systems.
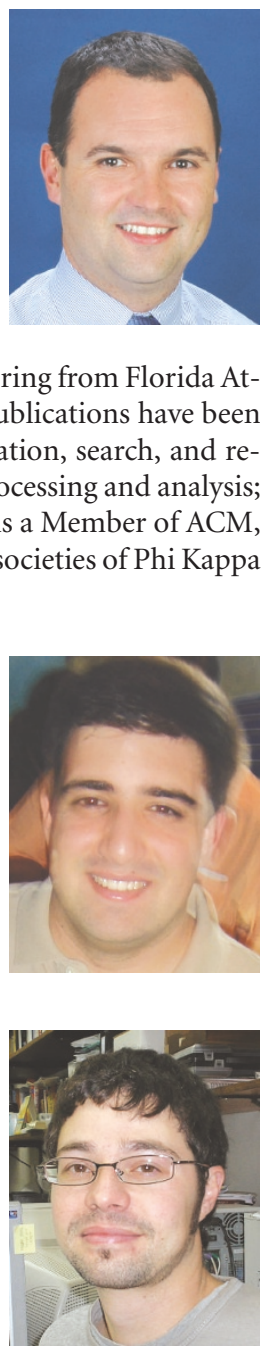

Humberto R. Gamba is a Lecturer in the Department of Electronics at Federal University of Technology Paraná (UTFPR) in Curitiba, Brazil. He received his B.S. degree in electrical engineering from UTFPR, a Master's degree in electrical engineering from Campinas State University (Campinas, Brazil) and a Ph.D. degree in medical physics from University of London. His current research interests include contentbased image retrieval, fMRI, image processing, and electronics instrumentation.

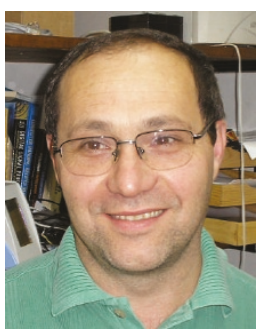

